\title{
Phosphatidylinositol 4,5-bisphosphate clusters act as molecular beacons for vesicle recruitment
}

\author{
Alf Honigmann ${ }^{1,8}$, Geert van den Bogaart ${ }^{2,3,8}$, Emilio Iraheta ${ }^{4}, \mathrm{H}$ Jelger Risselada ${ }^{5}$, Dragomir Milovanovic ${ }^{2}$, \\ Veronika Mueller $^{1}$, Stefan Müllar ${ }^{6}$, Ulf Diederichsen ${ }^{6}$, Dirk Fasshauer ${ }^{4}$, Helmut Grubmüller ${ }^{5}$, Stefan W Hell ${ }^{1}$, \\ Christian Eggeling ${ }^{1,7}$, Karin Kühnel ${ }^{2} \&$ Reinhard Jahn $^{2}$
}

\begin{abstract}
Synaptic-vesicle exocytosis is mediated by the vesicular $\mathrm{Ca}^{2+}$ sensor synaptotagmin-1. Synaptotagmin-1 interacts with the SNARE protein syntaxin-1 A and acidic phospholipids such as phosphatidylinositol 4,5-bisphosphate (PIP2). However, it is unclear how these interactions contribute to triggering membrane fusion. Using PC12 cells from Rattus norvegicus and artificial supported bilayers, we show that synaptotagmin- 1 interacts with the polybasic linker region of syntaxin-1 $\mathrm{A}$ independent of $\mathrm{Ca}^{2+}$ through PIP2. This interaction allows both $\mathrm{Ca}^{2+}$-binding sites of synaptotagmin-1 to bind to phosphatidylserine in the vesicle membrane upon $\mathrm{Ca}^{2+}$ triggering. We determined the crystal structure of the C2B domain of synaptotagmin-1 bound to phosphoserine, allowing development of a high-resolution model of synaptotagmin bridging two different membranes. Our results suggest that PIP2 clusters organized by syntaxin- 1 act as molecular beacons for vesicle docking, with the subsequent $\mathrm{Ca}^{2+}$ influx bringing the vesicle membrane close enough for membrane fusion.
\end{abstract}

Neuronal exocytosis is mediated by SNARE proteins. The vesicular SNARE synaptobrevin-2 binds to an acceptor SNARE complex consisting of SNAP-25 and syntaxin-1A in the plasma membrane (reviewed in refs. 1-4). During binding, a tight $\alpha$-helical bundle is formed that pulls the membranes together and overcomes the energy barrier for membrane fusion. Syntaxin-1A and SNAP-25 are not uniformly distributed in the plasma membrane but cluster in $\sim 70$-nm-sized membrane domains ${ }^{5-7}$. We recently showed that polyanionic PIP2 is the dominant inner-leaflet lipid in these membrane domains enriched in syntaxin- $1 \mathrm{~A}^{7}$. Furthermore, we showed that syntaxin-1A clustering is driven by electrostatic interactions between PIP2 and a juxtamembrane polybasic linker region of syntaxin-1A that connects the SNARE domain and the transmembrane helix $(260-K A R R K K-265)^{7}$. The significance of these syntaxin-PIP2 clusters for fast exocytosis, however, has remained unclear.

Synaptotagmin-1 acts as a main $\mathrm{Ca}^{2+}$ sensor of neuronal exocytosis (reviewed in refs. 2-4). It consists of an N-terminal unstructured luminal domain, a single transmembrane helix and a cytoplasmic domain that contains a 61-residue cytoplasmic linker connecting the transmembrane domain to two calcium-binding $\mathrm{C} 2$ domains, called $\mathrm{C} 2 \mathrm{~A}$ and $\mathrm{C} 2 \mathrm{~B}$. C2A and $\mathrm{C} 2 \mathrm{~B}$ bind three and two $\mathrm{Ca}^{2+}$ ions, respectively, with affinities ranging from 0.1 to $2 \mathrm{mM}^{2,3}$. Despite researchers' intense efforts, how synaptotagmin- 1 triggers $\mathrm{Ca}^{2+}$-invoked exocytosis is still controversial, although the mechanism of action is likely to involve interactions of synaptotagmin-1 with both SNARE proteins and membranes. Synaptotagmin-1 binds SNAP-25, syntaxin-1A and the binary and ternary SNARE complexes (reviewed in refs. 2-4,8). Synaptotagmin-1 interactions with SNAREs are observable in the absence of $\mathrm{Ca}^{2+}$ (that is, with an excess of the $\mathrm{Ca}^{2+}$ chelator EGTA) and are enhanced by $\mathrm{Ca}^{2+}$. It has long been unclear how synaptotagmin-1 precisely binds SNARE proteins (reviewed in ref. 2), especially because regions of synaptotagmin-1 that were implicated in SNARE binding are also involved in and compete with membrane binding 9 . These discrepancies were recently resolved by a single-molecule study in which synaptotagmin-1 was proposed to interact only loosely with the SNARE complex through weak electrostatic interactions in a configuration that left the lipid-binding domains free for interactions with membranes ${ }^{8}$. A recent EPR study lent further support to a notvery-structured but rather dynamic binding of synaptotagmin-1 to the SNAREs ${ }^{10}$. Nevertheless, there are still some unresolved questions concerning the binding of synaptotagmin to SNAREs. For instance, an unstructured and dynamic binding mode is difficult to reconcile with the reported ability of synaptotagmin- 1 to replace complexin-1 from the SNARE complex ${ }^{11,12}$, and it does not explain the $\mathrm{Ca}^{2+}$ dependency of SNARE binding (reviewed in refs. 2-4,8). Binding of synaptotagmin-1 to membranes containing anionic phospholipids is better understood. In the absence of $\mathrm{Ca}^{2+}$, a conserved polybasic lysine stretch located on the $\mathrm{C} 2 \mathrm{~B}$ domain and other surface-exposed basic residues interact with anionic phospholipids such as monovalent phosphatidylserine and polyvalent PIP2. In the presence of $\mathrm{Ca}^{2+}$,

${ }^{1}$ Department of Nanobiophotonics, Max Planck Institute for Biophysical Chemistry, Göttingen, Germany. ${ }^{2}$ Department of Neurobiology, Max Planck Institute for Biophysical Chemistry, Göttingen, Germany. ${ }^{3}$ Department of Tumor Immunology, Radboud University Nijmegen Medical Centre, Nijmegen, The Netherlands. ${ }^{4}$ Faculty of Biology and Medicine, University of Lausanne, Lausanne, Switzerland. ${ }^{5}$ Department of Theoretical and Computational Biophysics, Max Planck Institute for Biophysical Chemistry, Göttingen, Germany. ${ }^{6}$ Institute for Organic and Biomolecular Chemistry, Georg-August-Universität Göttingen, Göttingen, Germany. ${ }^{7}$ Weatherall Institute of Molecular Medicine, University of Oxford, Oxford, UK. ${ }^{8}$ These authors contributed equally to this work. Correspondence should be addressed to R.J. (r.jahn@gwdg.de). 
a

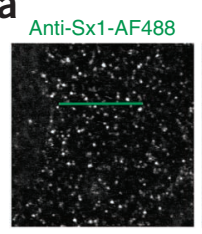

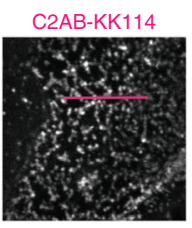

b

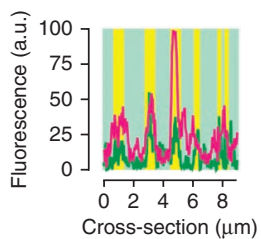

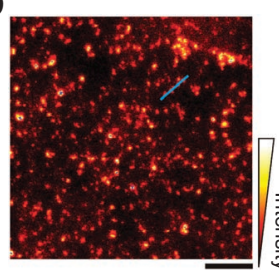

Figure 1 Synaptotagmin-1 binds syntaxin- 1 clusters in inverted sheets of PC12 cellderived plasma membranes. (a) Representative confocal images of unfixed membrane sheets showing immunolabeled syntaxin-1 (antiSx1-AF488; green) and the bound C2AB fragment of synaptotagmin-1, labeled with the fluorescent dye KK114 (100 nM C2ABKK114; magenta), in the absence of $\mathrm{Ca}^{2+}$. Right, fluorescence intensity profiles through the lines marked in the images (in counts per pixel; yellow bars, clustered regions). (b) Representative STED image of C2AB-KK114 on an unfixed PC12 membrane sheet. (c) Fluorescence intensity profiles through the line marked in $\mathbf{b}$, showing size and density of C2AB clusters. (d) Size distribution of the C2AB and syntaxin-1A clusters as determined from STED microscopy. Green, syntaxin-1A, diameter $80 \pm 23 \mathrm{~nm}$ (FWHM \pm s.d.), similar to that reported previously ${ }^{5}$; magenta, C2AB, diameter $75 \pm 26 \mathrm{~nm}$ (FWHM \pm s.d.). The distribution of the FWHM of 150 domains from six sheets and two independent preparations is shown. (e) Distribution of C2AB-KK114 without $\mathrm{Ca}^{2+}$ (left, purple), after addition of $200 \mu \mathrm{M} \mathrm{Ca}{ }^{2+}$ (middle, blue) or after chelation of previously added $\mathrm{Ca}^{2+}$ by addition of EGTA (orange, right). Plot at right shows fluorescence intensity profiles through the lines marked in the images (in counts per pixel; yellow bars, clustered regions). (f,g) Fluorescence signal in the areas between (f) and at (g) puncta, as determined from the images of C2AB-KK114; a.u., arbitrary units. Peak counts \pm range from six sheets of three independent preparations are shown; ${ }^{*} P=6 \times 10^{-4}$ $8 \times 10^{-4}$ by two-sided $t$ test. (Supplementary Fig. $1 \mathrm{c}$ shows distributions of C2AB binding to the puncta.) Scale bars throughout figure, $2 \mu \mathrm{m}$.

both $\mathrm{Ca}^{2+}$-binding sites of the $\mathrm{C} 2$ domains interact with anionic phospholipids ${ }^{2-4,10,13-27}$. In the $\mathrm{Ca}^{2+}$-bound state, the $\mathrm{C} 2$ domains of synaptotagmin-1 are able to tether two membranes ${ }^{17-20,22,24}$, but how exactly the C2 domains are able to simultaneously bind two membranes and whether such tethering is also relevant in the activation mechanism of synaptotagmin is still unclear. We performed a series of controlled binding experiments to resolve the interactions of synaptotagmin-1 with syntaxin-1 as well as with the vesicular and plasma membranes.

\section{RESULTS}

\section{Synaptotagmin-1 binds syntaxin-1A through PIP2}

Because synaptotagmin-1 binds both PIP2 and syntaxin-1A, we tested whether synaptotagmin-1 can also bind membrane clusters that are enriched in both syntaxin-1A and PIP2 (refs. 6,7). Indeed, a bacterially expressed soluble domain of synaptotagmin-1 (called $\mathrm{C} 2 \mathrm{AB}$ domain; residues $97-421$ ) bound $>80 \%$ of the syntaxin-1A clusters on inverted plasma-membrane sheets of PC12 cells (a neuroendocrine cell line) irrespective of $\mathrm{Ca}^{2+}$ (Fig. 1a). In these experiments, freshly prepared (nonfixed) membrane sheets were briefly ( $<5 \mathrm{~min}$ ) incubated with a monoclonal antibody to syntaxin-1, HPC-1, labeled with Alexa Fluor 488 (anti-Sx1-AF488), and with C2AB labeled at position S342C with the far-red fluorophore KK114. Super-resolution stimulated emission depletion (STED) microscopy with HPC-1 and a fluorescently labeled secondary antibody on fixed membrane sheets showed that the $\mathrm{C} 2 \mathrm{AB}$ clusters and syntaxin-1A membrane domains overlapped and were of similar size ( 75 nm; Fig. 1b-d and Supplementary Fig. 1a,b). Addition of $\mathrm{Ca}^{2+}$ to a final concentration of $200 \mu \mathrm{M}$ increased binding of $\mathrm{C} 2 \mathrm{AB}$ to the syntaxin-1A clusters and strongly increased $\mathrm{C} 2 \mathrm{AB}$ binding to the areas between the clusters (Fig. 1e-g and Supplementary Fig. 1c). To probe the mobility of membrane-bound $\mathrm{C} 2 \mathrm{AB}$ under these conditions, we applied fluorescence correlation spectroscopy in combination with STED (STED-FCS) ${ }^{28}$. STED-FCS allowed determination of the apparent diffusion coefficient $D$ for different observation areas ranging from 50 to $250 \mathrm{~nm}$ (full width at half-maximum intensity (FWHM)), revealing hindered diffusion in case of any deviation from a constant dependence $D(\mathrm{FWHM})^{28,29}$. As expected ${ }^{29}$, a fluorescent lipid phosphatidylcholine analog (Atto647N-PC) showed free Brownian diffusion with constant values $D=0.5 \mu \mathrm{m}^{2} \mathrm{~s}^{-1}$ (Fig. 2a). In contrast, the dynamics of $\mathrm{C} 2 \mathrm{AB}$ bound to the syntaxin-1A clusters were too slow to be evaluated by point FCS measurements because the clusterbound population strongly photobleached during a typical excitation period of $10 \mathrm{~s}$. C2AB bound to the areas between the clusters was mobile, but diffusion was strongly anomalous (that is, the measured diffusion times showed a nonlinear response when the observation area was reduced with STED), indicating hindered diffusion of the molecule (Fig. 2a). The decrease of $D$ for small FWHM reveals transient binding (trapping) of $\mathrm{C} 2 \mathrm{AB}$ to slow or immobilized membrane constituents and was observed previously for lipids with large polar head groups ${ }^{29}$, which indicated that we probed the diffusion of a C2AB-PIP2 complex. Indeed, a fluorescent PIP2 analog (Top-Fluor PIP2) partitioned to the same membrane clusters where C2AB bound (Fig. 2b,c). Moreover, overexpression of the phosphatase domain of synaptojanin-1 fused to a CAAX box to target it to the plasma membrane ${ }^{30}$ strongly reduced both $\mathrm{Ca}^{2+}$-independent and $\mathrm{Ca}^{2+}$-dependent C2AB binding to PC12 membrane sheets (Supplementary Fig. 2). Synaptojanin-1 is a polyphosphoinositide 5-phosphatase, and its expression results in complete removal of PIP2 from the plasma membrane $^{30}$ and dispersion of the syntaxin- 1 membrane domains ${ }^{7}$. Thus, synaptotagmin-1 binds selectively to the PIP2-syntaxin-1 clusters at the plasma membrane, and this raises the question of which parts of synaptotagmin-1 (C2AB) are involved in binding these clusters.

$\mathrm{C} 2 \mathrm{AB}$ mutants disrupted in $\mathrm{Ca}^{2+}$ binding to either the C2A (D178A D230A D232A) or C2B domain (D309A D363A D365A) were still able to bind the syntaxin-1A clusters on PC12 membrane sheets (Fig. 3a). However, $\mathrm{Ca}^{2+}$-dependent binding of mutant $\mathrm{C} 2 \mathrm{AB}$ to the areas between the domains was substantially reduced compared to that of wild-type. These results are probably explained by reduced $\mathrm{Ca}^{2+}$-dependent membrane binding in which the $\mathrm{Ca}^{2+}$-binding sites of the $\mathrm{C} 2 \mathrm{~A}$ and $\mathrm{C} 2 \mathrm{~B}$ domains insert in the membrane in a cooperative manner ${ }^{13,21}$. In sharp contrast to the $\mathrm{Ca}^{2+}$-binding mutants, $\mathrm{C} 2 \mathrm{AB}$ 
Figure 2 Synaptotagmin-1 binding to syntaxin-1 clusters is mediated by PIP2. (a) Dependence of the apparent diffusion coefficient $D$ on the FWHM of the observation area obtained by STED-FCS of C2AB-KK114 (C2AB; red) and PC-Atto647N (PC; blue) on PC12 membrane sheets in the presence of $200 \mu \mathrm{M} \mathrm{Ca}^{2+}$, indicating free diffusion for the $\mathrm{PC}$ and transient molecular interactions for the C2AB fluorescent analogs. Error bars, s.e.m. from four sheets. (b) Confocal images of unfixed PC12 membrane sheets preincubated with Top-Fluor PIP2 to localize PIP2-clusters (green) followed by incubation with labeled synaptotagmin-1 (100 nM C2AB-KK114; magenta), with $200 \mu \mathrm{M} \mathrm{Ca}{ }^{2+}$ (top) or after chelation of $\mathrm{Ca}^{2+}$ with an excess of EGTA (bottom). Scale bar, $2 \mu \mathrm{m}$. (c) Fluorescence intensity profiles through the lines marked in $\mathbf{b}$ (as in Fig. 1), indicating binding of C2AB to the PIP2 clusters in the absence of Ca ${ }^{2+}$ with two lysines of the polybasic lysine stretch converted to alanines (K326A K327A; the KAKA mutant ${ }^{15}$ ) no longer bound the syntaxin clusters in the absence of $\mathrm{Ca}^{2+}$ (Fig. 3a). However, again in contrast to the $\mathrm{Ca}^{2+}$-binding mutants, this mutant was still capable of $\mathrm{Ca}^{2+}$ dependent binding to the syntaxin-1A domains and the areas between the syntaxin-1A domains. These findings clearly indicate that the polybasic lysine stretch of synaptotagmin-1 binds the syntaxin-1A clusters through PIP2, and syntaxin-1 and synaptotagmin-1 may interact with one or more PIP2 molecules simultaneously (Fig. 3b), or alternatively PIP2 may unbind from syntaxin-1 before binding the C2B domain. Synaptotagmin-1 binding to syntaxin-1A through PIP2 is in line with competitive-inhibition experiments of SNARE binding by inositol hexabisphosphate ${ }^{16,31}$. Of course, we cannot exclude other modes of $\left(\mathrm{Ca}^{2+}\right.$-mediated) binding of synaptotagmin-1 to SNAREs, such as to SNAP-25 or to the anionic surface of binary or ternary SNARE complexes ${ }^{2,3,8,10}$, but apparently these binding sites are not easily accessible when the SNAREs are in a native environment.

We recently demonstrated the reconstitution of PIP2-syntaxin-1A clusters in artificial membranes ${ }^{7}$. Here we studied binding of synaptotagmin-1 to these clusters by using supported lipid bilayers. The main advantages of supported lipid bilayers are that they allow precise control of the lipid composition and are easy to image by fluorescence microscopy. We prepared stacked bilayers of two or three membranes by spin-coating lipid mixtures in chloroform on microscope cover glasses. Artifacts due to electrostatic and hydrophobic interactions with the glass support can be circumvented by studying lipid bilayers that are on top of the basal bilayer ${ }^{32}$. PIP2-syntaxin-1A membrane clusters were reconstituted in membranes composed of
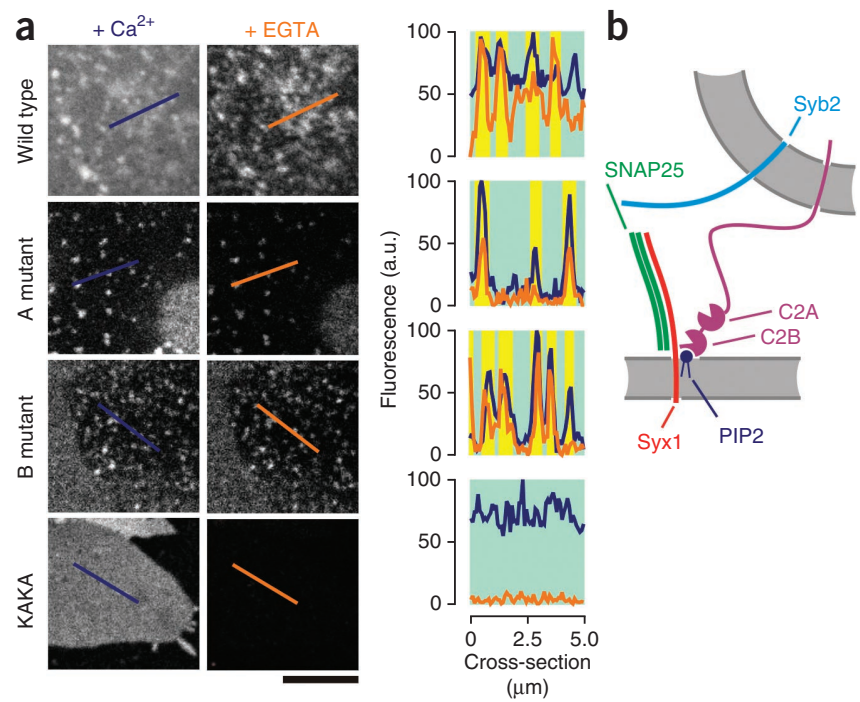

b

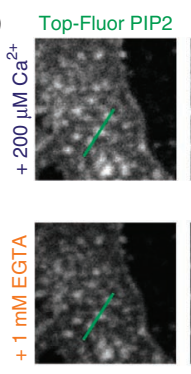

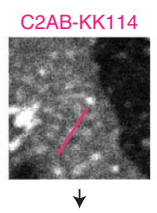

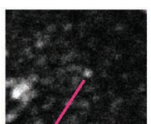

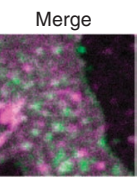

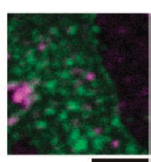

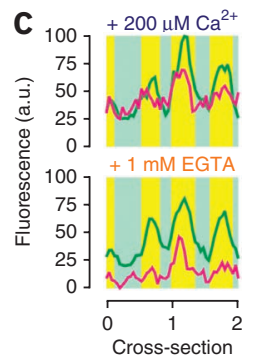

$(\mu \mathrm{m})$ 1,2-dioleoyl-sn-glycero-3-phosphatidylcholine (DOPC), with or without $20 \%$ phosphatidylserine, $30 \%$ cholesterol, 1-2\% PIP2 and syntaxin-1 $\mathrm{A}_{257-288}$ that consisted of a conserved polybasic juxtamembrane linker region and the C-terminal transmembrane helix of syntaxin-1A (Fig. 4a-d and Supplementary Fig. 3a). As shown previously $^{7}$, syntaxin-1 $\mathrm{A}_{257-288}$ clustering was dependent on the presence of PIP2 (Supplementary Fig. 3b). Moreover, PIP2-syntaxin-1 $A_{257-288}$ clusters were also present when an excess of phosphatidylserine or cholesterol was used (Supplementary Fig. 3c), and fluorescent TopFluor PIP2 was enriched at the syntaxin-1 $\mathrm{A}_{257-288}$ clusters (Fig. 4e,f and Supplementary Fig. 3d). Similarly (Fig. 1), C2AB bound the PIP2-syntaxin-1 $\mathrm{A}_{257-288}$ clusters in the absence of $\mathrm{Ca}^{2+}$, and the addition of $\mathrm{Ca}^{2+}$ to a final concentration of $100-200 \mu \mathrm{M}$ increased $\mathrm{C} 2 \mathrm{AB}$ binding to both the puncta and the areas between the puncta (Fig. 4a-d and Supplementary Fig. 3a). Addition of the $\mathrm{Ca}^{2+}$ chelator ethylene glycol tetraacetic acid (EGTA) to a final concentration of $1 \mathrm{mM}$ only slightly affected the clusters but substantially removed $\mathrm{C} 2 \mathrm{AB}$ that was bound to the areas between the membrane clusters. In the absence of syntaxin-1 $\mathrm{A}_{257-288}$, C2AB bound homogeneously to membranes containing 2 mol\% PIP2, but only when $\mathrm{Ca}^{2+}$ was present (Fig. 5a). Apparently, this concentration of PIP2 was not high enough for substantial $\mathrm{C} 2 \mathrm{AB}$ binding in the absence of $\mathrm{Ca}^{2+}$, which required at least $5 \mathrm{~mol} \%$ PIP2 (Supplementary Fig. 3e). Control experiments without PIP2 and with $\mathrm{Mg}^{2+}$ showed that $\mathrm{C} 2 \mathrm{AB}$ binding to supported lipid bilayers was specific for both PIP2 and $\mathrm{Ca}^{2+}$ (Supplementary Fig. 3f,g).

To test the nature of synaptotagmin-1 binding to membrane clusters of PIP2 and syntaxin-1A, we performed experiments with short cationic peptides that mimic the polybasic linker region of syntaxin-1A. A peptide corresponding to the polybasic region of syntaxin-1 (260-KARRKK-265) was able to cluster C2AB in artificial membranes in the presence of PIP2 (Fig. 5a-d and Supplementary Fig. 4), but this clustering was not specific for the sequence of this region, as it was also induced by pentalysine. Membrane clustering by syntaxin$1 \mathrm{~A}_{260-265}$ was not observed when phosphatidylserine instead of PIP2 was used (Fig. 5e) and was relatively insensitive to $\mathrm{Ca}^{2+}$ or $\mathrm{Mg}^{2+}$

Figure 3 Synaptotagmin-1 binds the syntaxin-1 clusters through the conserved polybasic patch. (a) Representative confocal images of unfixed PC12 cell-membrane sheets incubated with $100 \mathrm{nM}$ of different Alexa Fluor 488-labeled C2AB mutants, either in the presence of $200 \mu \mathrm{M} \mathrm{Ca}^{2+}$ (blue) or after chelation of the $\mathrm{Ca}^{2+}$ with an excess of EGTA (orange). Right, fluorescence intensity profiles through the lines marked in the images (as in Fig. 1). A mutant and $\mathrm{B}$ mutant indicate disrupted $\mathrm{Ca}^{2+}$ binding sites in the $\mathrm{C} 2 \mathrm{~A}$ or $\mathrm{C} 2 \mathrm{~B}$ domains, respectively. (b) Model of $\mathrm{Ca}^{2+}$ independent binding of the basic stretch of synaptotagmin-1 (purple) to membrane domains enriched in PIP2 (blue) and syntaxin-1A (Syx1; red). Green, SNAP25; cyan, synaptobrevin-2 (Syb2). Scale bar, $5 \mu \mathrm{m}$. 
Figure 4 Synaptotagmin-1 binding to PIP2-syntaxin-1A clusters in artificial stacked supported membranes generated by spincoating on glass surfaces. (a) Representative confocal images of membranes (69\% DOPC, $30 \%$ cholesterol and 1\% PIP2) containing syntaxin-1 $\mathrm{A}_{257-288}$ labeled with Atto647N (SxTMH; magenta), incubated with $100 \mathrm{nM}$ C2AB fragment labeled with Alexa Fluor 488 (C2AB-AF488; green) in the absence of $\mathrm{Ca}^{2+}$ (purple), after addition of $200 \mu \mathrm{M} \mathrm{Ca}^{2+}$ (blue) or after chelation of previously added $\mathrm{Ca}^{2+}$ with EGTA (orange). (b) Fluorescence intensity profiles through the lines marked in a (as in Fig. 1). (c,d) Fluorescence (peak counts) between (c) and at (d) puncta, as determined from the different images of C2AB-AF488

\pm range for six positions and three independent bilayers; ${ }^{*} P=0.006-0.01$ by two-sided $t$ test. (Supplementary Fig. 3a shows distributions of C2AB binding to the puncta.) (e,f) Same as a and $\mathbf{b}$ in the absence of $\mathrm{Ca}^{2+}$ but with fluorescent Top-Fluor PIP2 (0.1 mol\% TF-PIP2; green) instead of C2AB, showing PIP2 enrichment in the SxTMH clusters. Scale bars, $2 \mu \mathrm{m}$. a

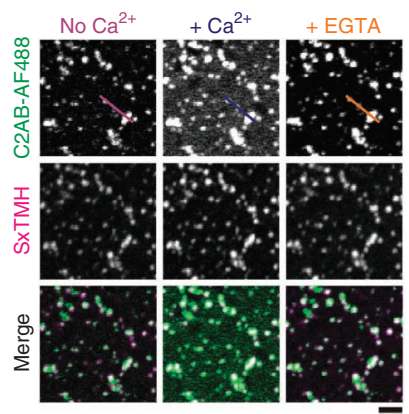

b

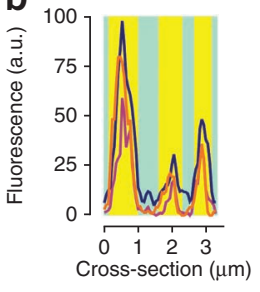

e
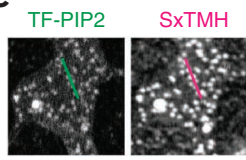

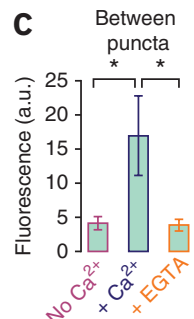

d

d Puncta

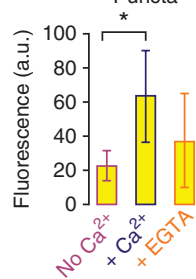

$f$ 雍

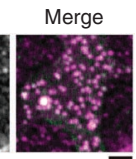

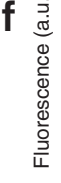

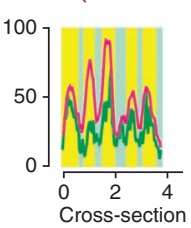

(um)

(Fig. 5 and Supplementary Fig. 4). PIP2 clustering by polyvalent cationic peptides has also been observed previously ${ }^{33} \cdot \mathrm{Ca}^{2+}$ is also known to cluster PIP2 (ref. 34), and indeed C2AB was clustered at high $\mathrm{Ca}^{2+}$ concentrations (above $0.5 \mathrm{mM}$ ) in the absence of the polybasic peptides (Supplementary Fig. 5). However, the clusters induced by high $\mathrm{Ca}^{2+}$ concentrations were different from the clusters induced by the basic peptides because only in the latter was phosphatidylcholine at least partially depleted from the clusters (Fig. 5a). Moreover, $\mathrm{Ca}^{2+}$ induced but not ligand-induced cluster formation was reversed when $\mathrm{Ca}^{2+}$ was subsequently chelated with EGTA (Supplementary Fig. 5). Altogether, we conclude that synaptotagmin-1 binds in a $\mathrm{Ca}^{2+}$ independent manner to membrane domains in which PIP2 is concentrated around the polybasic linker of syntaxin- $1 \mathrm{~A}^{7}$.

\section{Vesicle recruitment to PIP2-syntaxin-1A membrane clusters}

It is well established ${ }^{17-20,22,24}$ that the $\mathrm{C} 2 \mathrm{AB}$ fragment can cross-link two membranes in the presence of $\mathrm{Ca}^{2+}$. Therefore, we tested whether

a

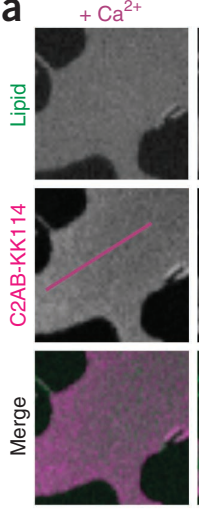

C
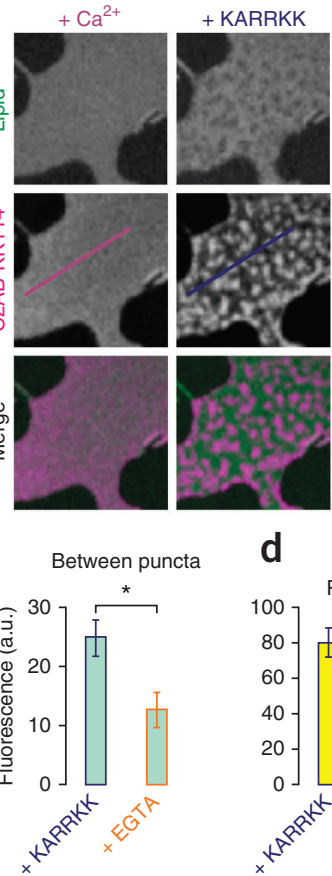

d
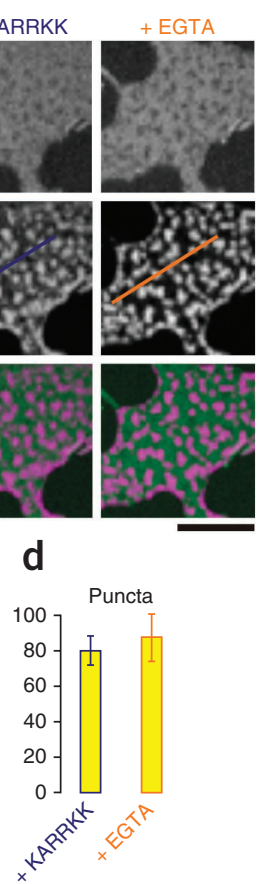

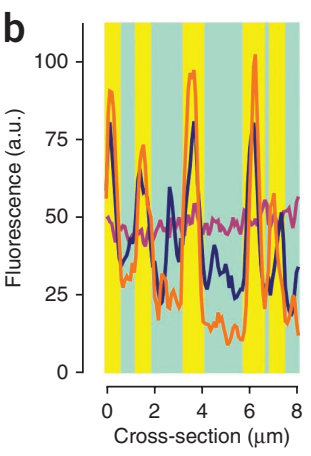

e

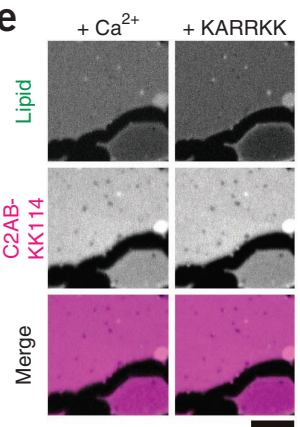

$\mathrm{C} 2 \mathrm{AB}$ can recruit vesicles to the PIP2-syntaxin-1A clusters. Indeed, in the presence of $\mathrm{C} 2 \mathrm{AB}$ we observed binding of 35 -nm-sized ${ }^{17}$ artificial liposomes (composed of a 5:2:1:1 molar ratio of DOPC/1, 2-dioleoyl-sn-glycero-3-phosphoethanolamine (DOPE)/1,2-dioleoyl$s n$-glycero-3-phosphatidylserine (DOPS)/cholesterol with $1 \%$ of the fluorescent lipid analog BODIPY-FL PC) to PC12 membrane sheets (Fig. 6a). Binding was $\mathrm{Ca}^{2+}$ dependent but was only partially reversed by EGTA, and we attribute this partial reversal by EGTA to shortrange (hydrophobic) interactions that occur after membrane binding. Liposome tethering was observed only when the liposomes contained $>5 \%$ of the anionic phospholipid DOPS (Fig. 6b). Tethering clearly overlapped with the sites where $\mathrm{C} 2 \mathrm{AB}$ was bound (Fig. 6a). However, some tethering (albeit weaker) was also observed in the absence of $\mathrm{C} 2 \mathrm{AB}$ (Fig. 6c). This was probably caused by endogenous synaptotagmin-1, because liposome tethering was substantially reduced by preincubation with antibodies specific for synaptotagmin-1 (Fig. 6d). To exclude interference by endogenous synaptotagmin-1, we performed experiments with supported lipid bilayers of precisely controlled composition (Fig. 7a,b and Supplementary Fig. 6). Liposome recruitment to reconstituted PIP2-syntaxin-1 clusters in artificial membranes was clearly dependent on the presence of the $\mathrm{C} 2 \mathrm{AB}$ fragment or of full-length synaptotagmin-1 incorporated in the liposomes, and it occurred irrespective of the presence of phosphatidylserine in the supported bilayer.

We used artificial supported lipid membranes and different synaptotagmin-1 mutants to determine which of the binding sites of synaptotagmin-1 are involved in liposome recruitment (Fig. $7 \mathbf{b}$ and

Figure 5 Synaptotagmin-1 binds PIP2 clusters induced by the polybasic linker region of syntaxin-1 in artificial membranes. (a) Representative confocal images of supported lipid bilayers (DOPC and 2\% PIP2) prelabeled with $0.1 \mathrm{~mol} \%$ of the fluorescent lipid analog BODIPY-FL PC (lipid; green) and then incubated with $100 \mathrm{nM} \mathrm{C2AB}$ labeled with KK114 (C2AB-KK114; magenta) in the presence of $200 \mu \mathrm{M} \mathrm{Ca}{ }^{2+}$ (control; purple, left), after addition of $20 \mu \mathrm{M}$ of the soluble syntaxin-1 $\mathrm{A}_{260-265}$ linker peptide (KARRKK; blue, middle) and after addition of EGTA (with the peptide still present; orange, right). (b) Fluorescence intensity profiles through the lines marked in a (as in Fig. 1). (c,d) Fluorescence peak counts between (c) and at (d) puncta, as determined from the confocal images of C2AB-KK114 \pm range for six positions and three independent bilayers; * $P=2 \times 10^{-3}$ by two-sided $t$ test. (Supplementary Fig. 4a shows distributions of $C 2 A B$ binding to the puncta.) (e) Control experiments as in a but using membranes containing phosphatidylserine instead of PIP2 (80\% DOPC and 20\% DOPS). Scale bars, $5 \mu \mathrm{m}$. 
Figure $6 \mathrm{Ca}^{2+}$-dependent recruitment of liposomes to the plasma membrane by synaptotagmin- 1 .

(a) Representative confocal images of unfixed PC12 membrane sheets incubated with BODIPY-labeled liposomes containing $11 \%$ phosphatidylserine (PS; green; $5 \mathrm{nM}$ final liposome concentration ${ }^{17}$ ) and $100 \mathrm{nM}$ C2AB labeled with KK114 (C2AB; magenta) in the absence of $\mathrm{Ca}^{2+}$ (no Ca ${ }^{2+}$; top) or after addition of $200 \mu \mathrm{M} \mathrm{Ca}^{2+}$ (+ $\mathrm{Ca}^{2+}$; bottom). Right, fluorescence intensity profiles (in counts per pixel; yellow bars, clustered regions) through the lines marked in the images (as in Fig. 1). (b) Same as a in the presence of $\mathrm{Ca}^{2+}$ but with liposomes lacking phosphatidylserine (no PS). (c) Same as a in the presence of $\mathrm{Ca}^{2+}$ but in the absence of $\mathrm{C} 2 \mathrm{AB}$. The membrane sheet was visualized with Atto647N-PE (lipid; magenta). (d) Same as a but with endogenous synaptotagmin-1 blocked with an Atto647N-labeled antibody (Anti-Syt1-A647N). Scale bars, $5 \mu \mathrm{m}$. a

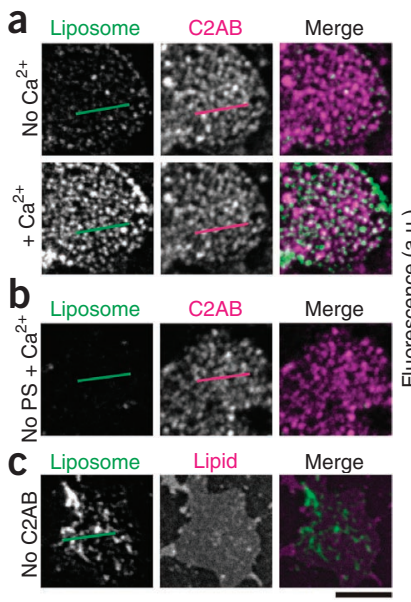

d

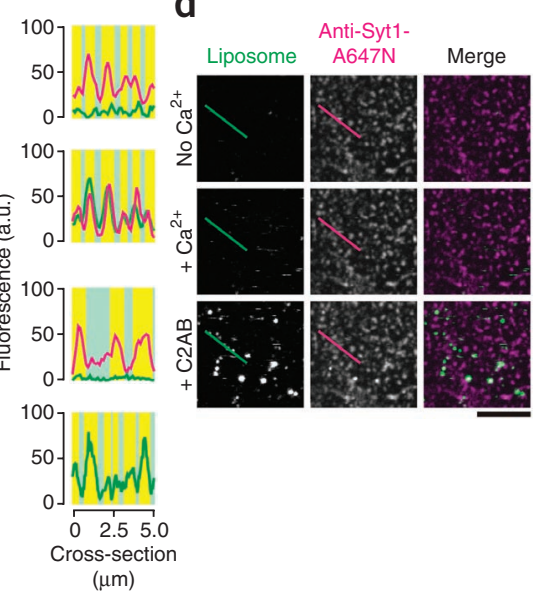

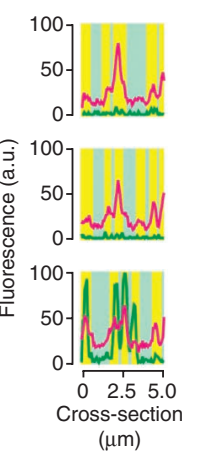

$(\mu \mathrm{m})$
Supplementary Fig. 6). Notably, liposome binding to the membrane was prevented when a mutant disrupted in $\mathrm{Ca}^{2+}$ binding to both $\mathrm{C} 2 \mathrm{~A}$ and C2B (AB mutant, D178A D230A D232A D309A D363A D365A) was used (Fig. 7b). In contrast, mutating only one of the $\mathrm{Ca}^{2+}$-binding sites (A mutant, D178A D230A D232A; or B mutant, D309A D363A D365A) or only the polybasic patch (KAKA mutant, K326A K327A) did not suffice to markedly reduce liposome tethering. The $\mathrm{C} 2 \mathrm{~B}$ domain (residues 271-421), but not the C2A domain (residues 96-263), was able to tether liposomes, in agreement with some earlier observations ${ }^{19}$ but not others ${ }^{20,22}$. Finally, liposome tethering was still observed with $\mathrm{C} 2 \mathrm{AB}$ with two conserved arginines mutated that are located opposite the $\mathrm{Ca}^{2+}$-binding loop (RQRQ mutant, R398Q R399Q). This mutant displays severely disrupted $\mathrm{Ca}^{2+}$-stimulated synchronous release in neurons ${ }^{12}$. Arg398 and Arg399 potentially interact in trans with anionic membranes ${ }^{19,24}$, although this is controversial, and no or only minor interactions with membranes have been reported ${ }^{20,27}$. In fact, Arg398 and Arg399 have recently been proposed to cross-link membranes through oligomerization of the $\mathrm{C} 2 \mathrm{AB}$ fragment, although this oligomerization required higher concentrations of $\mathrm{C} 2 \mathrm{AB}$ than the $100 \mathrm{nM}$ used in our experiments ${ }^{20}$. Accordingly, we found no indication for such oligomerization of $\mathrm{C} 2 \mathrm{AB}$ under our experimental conditions because (i) liposomes
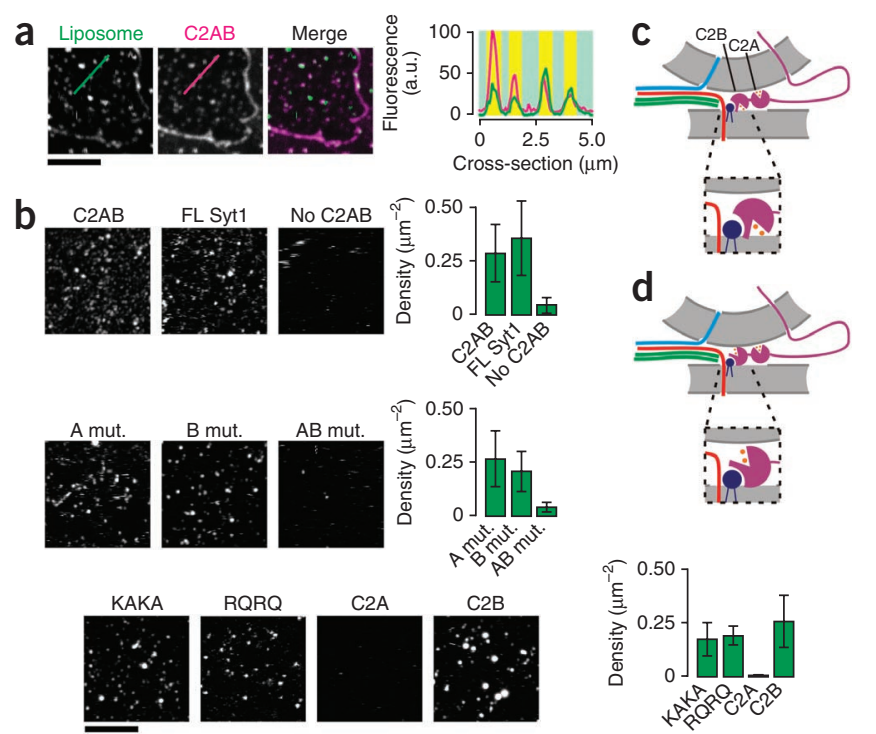

could still be tethered when $\mathrm{C} 2 \mathrm{AB}$ was prebound to the supported lipid bilayers and unbound $\mathrm{C} 2 \mathrm{AB}$ was removed before addition of the liposomes (Supplementary Fig. 7a), (ii) we did not find evidence for oligomerization by fluorescence cross-correlation spectroscopy when using a mixture of $\mathrm{C} 2 \mathrm{AB}$ labeled with different dyes, regardless of the presence of $\mathrm{Ca}^{2+}$ or membranes (Supplementary Fig. 7b), and (iii) the RQRQ mutant (with impaired oligomerization ${ }^{20}$ ) was still capable of liposome tethering (Fig. 7 b).

Together, these data suggest that the $\mathrm{C} 2 \mathrm{AB}$ fragment can tether liposomes in at least two possible conformations. In the first conformation, the $\mathrm{C} 2 \mathrm{~B}$ domain is oriented parallel to the membrane, with both the polybasic lysine stretch and the $\mathrm{Ca}^{2+}$-binding site of the C2B domain interacting with the plasma membrane, leaving Arg398, Arg399 (refs. 19,24) and the C2A domain free to interact in trans with the vesicle membrane (Fig. 7c). This first configuration would explain the capability of the KAKA mutant to tether liposomes (Fig. 7b). In the second conformation, the $\mathrm{C} 2 \mathrm{~B}$ domain is oriented perpendicularly to the plasma membrane, and the polybasic patch interacts with the plasma membrane, whereas both $\mathrm{Ca}^{2+}$-binding sites interact with the vesicular membrane (Fig. 7d). This second configuration would explain the capability of the mutants with the $\mathrm{Ca}^{2+}$-binding sites of either (but not both) the $\mathrm{C} 2 \mathrm{~A}$ or $\mathrm{C} 2 \mathrm{~B}$ domains disrupted to tether

Figure 7 Two membrane binding modes of synaptotagmin-1.

(a) Representative confocal images of supported lipid bilayers (lipid composition as in Fig. 5) containing unlabeled syntaxin-1 $A_{257-288}$ and incubated with BODIPY-labeled liposomes (liposome; green; $5 \mathrm{nM}$ liposome concentration ${ }^{17}$ ) and $100 \mathrm{nM}$ C2AB-KK114 (C2AB; magenta) in the presence of $200 \mu \mathrm{M} \mathrm{Ca}^{2+}$. (b) Representative confocal images of BODIPYlabeled liposomes bound to supported lipid bilayers with a more physiological composition (52\% DOPC, 15\% DOPS, 3\% PIP2 and 30\% cholesterol) and for different unlabeled C2AB mutants. C2AB, wild-type C2AB; FL Syt1, 1:1,000 protein/lipid ratio of full-length synaptotagmin-1 incorporated in the liposomes; no $C 2 A B$, absence of $C 2 A B ; A$ mut., C2A mutant; $B$ mut., C2B mutant; $A B$ mut., double mutant; KAKA, KAKA mutant; RQRQ, RQRQ mutant; $C 2 A$, $\mathrm{C} 2 \mathrm{~A}$ domain; $\mathrm{C} 2 \mathrm{~B}, \mathrm{C} 2 \mathrm{~B}$ domain. The bar graphs indicate the liposome binding efficiency and show the membrane-bound liposome densities determined for eight different regions from at least two independent experiments ( \pm range). (Supplementary Fig. 6 shows distributions of liposome binding to the puncta.) As shown, no binding was detected in the absence of $C 2 A B$, with the double mutant or with the $\mathrm{C} 2 \mathrm{~A}$ domain. (c) Model of vesicle recruitment by synaptotagmin- 1 in the presence of $\mathrm{Ca}^{2+}$ (orange) in which the $\mathrm{C} 2 \mathrm{~B}$ domain is oriented in a parallel orientation, with the $\mathrm{Ca}^{2+}$-binding site interacting with the plasma membrane. Color coding is as in Figure $\mathbf{3 b}$. (d) Same as $\mathbf{c}$ but with the $\mathrm{C} 2 \mathrm{~B}$ domain in a perpendicular orientation, with the $\mathrm{Ca}^{2+}$-binding site of $\mathrm{C} 2 \mathrm{~B}$ interacting with the vesicular membrane. Scale bars, $5 \mu \mathrm{m}$. 
a

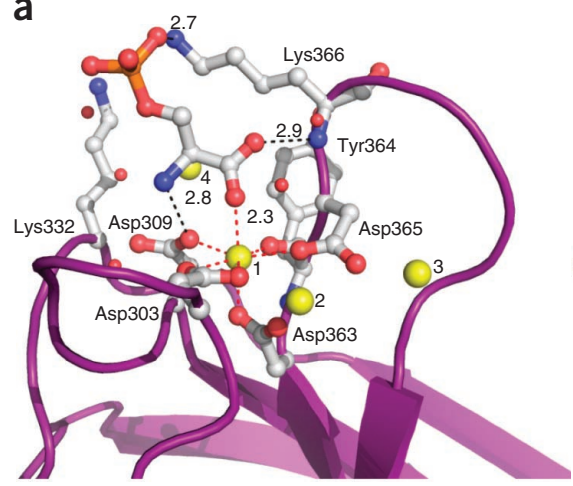

C

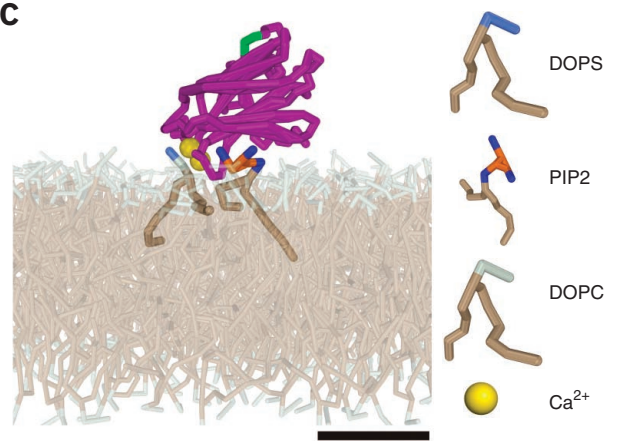

b
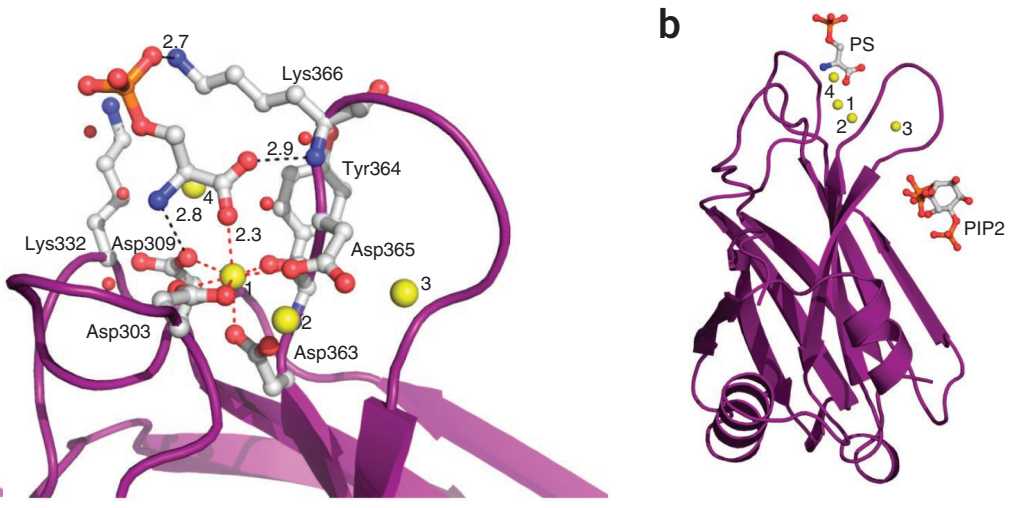

d

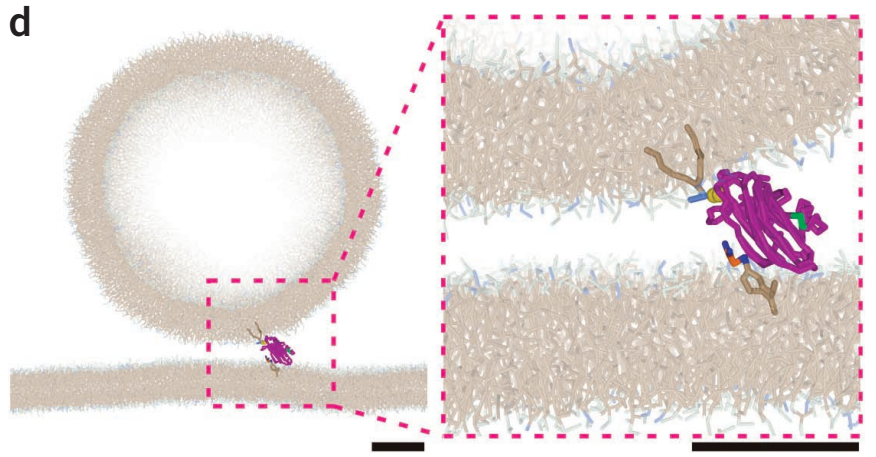

Figure 8 Lipid binding modes of the C2B domain. (a) Stereo view showing a close-up of the phosphoserine-bound $\mathrm{Ca}^{2+}$-binding site of the $\mathrm{C} 2 \mathrm{~B}$ domain from synaptotagmin-1. Coordination of calcium 1 is shown with red dashed lines, and the interactions of phosphoserine with the protein are marked with black dashed lines. Calcium ions are drawn as yellow spheres. Distances are given in angstroms. (b) Overall structure of the C2B fragment with the four bound $\mathrm{Ca}^{2+}$ ions and phosphoserine (PS). We modeled the head group of PIP2 into the polybasic stretch on the basis of PDB $3 G P E 39$.

(c) Snapshot from a coarse-grained molecular dynamics simulation run of the parallel membrane-binding conformation of the C2B domain. Here, both the phosphatidylserine-interacting $\mathrm{Ca}^{2+}$-binding site and the PIP2-interacting polybasic stretch interact with the same membrane, as shown by EPR ${ }^{24}$. Arg398 and Arg399 are shown in green. Scale bar, $2 \mathrm{~nm}$. (d) Snapshot of membrane cross-linking by the C2B domain (in perpendicular conformation), showing perturbation of the $\mathrm{C} 2 \mathrm{~B}$ fragment in the lower membrane (Supplementary Fig. 9). The membranes were composed of $80 \%$ DOPC and 20\% DOPS. Scale bars, $5 \mathrm{~nm}$.

liposomes (Fig. 7b). The second conformation seems unexpected, given the close proximity of the polybasic patch and the $\mathrm{Ca}^{2+}$-binding site on the C2B domain. Indeed, it was concluded from EPR measurements that the $\mathrm{C} 2 \mathrm{~B}$ domain bound in a parallel orientation, and the presence of PIP2 resulted only in the tilting of the C2B domain toward the membrane ${ }^{24}$. However, the membrane-bound orientation of the structurally similar C2 domain of protein kinase $\mathrm{C} \alpha(\mathrm{PKC} \alpha)$ is still under debate, and both parallel ${ }^{35,36}$ and perpendicular ${ }^{37}$ binding have been reported. Accordingly, although it is generally agreed that PIP2 changes the membrane orientation of $\mathrm{C} 2-\mathrm{PKC} \alpha$ relative to the membrane, it is debated whether $\mathrm{C} 2-\mathrm{PKC} \alpha$ tilts toward the membrane $^{35}$ (similarly to $\mathrm{C}_{2} \mathrm{~B}^{24}$ ) or away from the membrane ${ }^{36}$. Our data now indicate that the $\mathrm{C} 2 \mathrm{~B}$ membrane can tether liposomes in both parallel and perpendicular conformations.

Membrane tethering of the $\mathrm{C} 2 \mathrm{AB}$ domain in a perpendicular conformation would imply that $\mathrm{C} 2 \mathrm{~B}$ interacts with both the plasma membrane and the vesicular membrane simultaneously. It seems logical that the polybasic patch of synaptotagmin-1 would interact with the plasma membrane because PIP2 is the dominant innerleaflet lipid in the plasma membrane at the sites of docked vesicles $^{7}$, whereas it is believed to be absent from synaptic vesicles. The polybasic stretch is a conserved PIP2-binding motif, as shown previously for the $\mathrm{C} 2$ domains of rabphilin-3A and $\mathrm{PKC} \alpha^{38,39}$ and in the recent NMR structure of synaptotagmin-1 with inositol 6-phosphate ${ }^{25}$. Our findings indicate that the $\mathrm{Ca}^{2+}$-binding site on the $\mathrm{C} 2 \mathrm{~B}$ domain can interact with the vesicular membrane because a $\mathrm{C} 2 \mathrm{AB}$ mutant disrupted in $\mathrm{Ca}^{2+}$ binding still binds the PIP2-syntaxin-1A membrane clusters but no longer can tether liposomes. These interactions of the $\mathrm{Ca}^{2+}$-binding pocket may occur through phosphatidylserine or PIP2, which can complete the $\mathrm{Ca}^{2+}$-coordination site and increase the $\mathrm{Ca}^{2+}$-binding affinity of synaptotagmin-1, as is well described in the literature $13,18,27,40$.

Simultaneous interactions of C2B with phosphatidylserine and PIP2 To shed more light on how binding of the two adjacent sites to two opposing membranes can be accommodated, we crystallized the $\mathrm{C} 2 \mathrm{~B}$ fragment in the presence of phosphoserine as an analog for phosphatidylserine and determined the structure at 1.5 - $\AA$ resolution (Fig. 8a,b, Supplementary Fig. 8a and Table 1). Crystals had two molecules in the asymmetric unit, and both contained phosphoserine. The carboxyl group of phosphoserine is bound to a $\mathrm{Ca}^{2+}$ atom (calcium 1), which explains the $\mathrm{Ca}^{2+}$ dependency of phosphatidylserine binding. Binding of the phosphoserine carboxyl group completes the (pentagonal bipyramidal) coordination site of calcium 1 (Fig. 8a). In the apo structure (PDB 1UOV), this site is occupied by a water molecule ${ }^{41}$. Phosphoserine is further stabilized by extensive interactions with the protein, involving all of its functional groups. The amine group of phosphoserine forms a hydrogen bond with the carboxyl group of Asp309, and the phosphoserine carboxyl group forms a hydrogen bond with the amide nitrogen of Lys366 (Fig. 8a). Lys366 also participates in a salt bridge with the phosphoserine phosphate group, which is surface exposed. In the crystal 
Table 1 Data collection and refinement statistics

\begin{tabular}{|c|c|}
\hline & C2B with PS \\
\hline \multicolumn{2}{|l|}{ Data collection ${ }^{a}$} \\
\hline Space group & $P 42_{1} 2$ \\
\hline \multicolumn{2}{|l|}{ Cell dimensions } \\
\hline$a, b, c(\AA)$ & $97.0,97.0,89.0$ \\
\hline Resolution $(\AA)$ & $50-1.50(1.54-1.50)^{\mathrm{b}}$ \\
\hline$R_{\mathrm{sym}}(\%)$ & 3.5 (25.9) \\
\hline$I / \sigma I$ & $43.9(7.9)$ \\
\hline Completeness (\%) & $97.7(81.7)$ \\
\hline Redundancy & $12.2(7.4)$ \\
\hline Wilson $B$ factor $\left(\AA^{2}\right)$ & 13.7 \\
\hline \multicolumn{2}{|l|}{ Refinement } \\
\hline Resolution $(\AA \AA)$ & $50-1.50$ \\
\hline No. reflections & 66,840 \\
\hline$R_{\text {work }} / R_{\text {free }}$ & $18.3 / 20.4$ \\
\hline \multicolumn{2}{|l|}{ No. atoms } \\
\hline Protein & 2,413 \\
\hline Ligand (2 PS)/ions (7 $\left.\mathrm{Ca}^{2+}, 5 \mathrm{SCN}^{-}\right)$ & $22 / 22$ \\
\hline Water & 256 \\
\hline \multicolumn{2}{|l|}{$B$ factors $\left(\AA^{2}\right)$} \\
\hline Overall & 13.5 \\
\hline Protein & 12.6 \\
\hline Ligand (2 PS)/ions ( $\left.\mathrm{Ca}^{2+}, \mathrm{SCN}^{-}\right)$ & PS, $14.6 / \mathrm{Ca}^{2+}, 11.8 / \mathrm{SCN}^{-}, 14.4$ \\
\hline Water & 21.6 \\
\hline \multicolumn{2}{|l|}{ r.m.s. deviations } \\
\hline Bond lengths $(\AA)$ & 0.009 \\
\hline Bond angles $\left({ }^{\circ}\right)$ & 1.23 \\
\hline
\end{tabular}

PS, phosphoserine.

aData were collected from a single crystal. bValues in parentheses are for highest-resolution shell.

structure, Lys332 is involved in crystal-packing contacts by forming a salt bridge with Glu350 from a symmetry-related molecule and is too far away to form a salt bridge with the phosphate group $(6.5 \AA)$ but might otherwise swing in and interact with the phosphate group. Both Lys332 and Lys366 are structurally conserved and correspond to Arg198 and Arg233 in the C2A domain. Of note, the interaction between phosphoserine and the C2B fragment is completely different from the interaction described previously between phosphatidylserine and the C2 domain of PKC $\alpha$ (Supplementary Fig. 8b) ${ }^{40}$. In the PKC $\alpha$ structure, the phosphate group of phosphatidylserine interacts with calcium 1, and the phosphatidylserine carboxyl group forms a hydrogen bond to the amide nitrogen of Asn189 instead. In contrast to the sparse interactions of the phosphatidylserine head group observed in the PKC $\alpha$ structure, phosphoserine interacts extensively with $\mathrm{C} 2 \mathrm{~B}$ in our structure. We observed three and four calcium atoms bound in the two molecules of the asymmetric unit, whereas typically two bound calcium ions are reported for $\mathrm{C} 2 \mathrm{~B}^{26,41}$ (further discussed in Supplementary Fig. 8c).

To better understand how the $\mathrm{C} 2 \mathrm{~B}$ domain can bind simultaneously to the PIP2-containing plasma membrane and the phosphatidylserinecontaining vesicle membrane, we used the crystal structure of the C2 domain of PKC $\alpha$ with the head group of PIP2 (ref. 39) to model $\mathrm{PIP} 2$ to the polybasic patch in our new $\mathrm{Ca}^{2+}$ - and phosphatidylserinebound $\mathrm{C} 2 \mathrm{~B}$-domain structure (Fig. 8b). We then used this structure as a starting point for carrying out molecular dynamics simulations. In these coarse-grained simulations, several atoms were represented by one simulation bead ${ }^{17,42}$, which allowed for simulations of relatively large systems of $>5,000$ lipids. For the parallel binding mode
(Fig. 8c), we incorporated both lipids into a membrane composed of $80 \%$ DOPC and $20 \%$ DOPS. For the perpendicular binding mode (Fig. 8d), we incorporated PIP2 in a planar membrane (that is, mimicking the plasma membrane) and the phosphatidylserine in a vesicle of $40-\mathrm{nm}$ size (that is, mimicking a synaptic vesicle). Both systems were stable over simulation times of $500 \mathrm{~ns}-1 \mu \mathrm{s}$, which supports our conclusion that both conformations of interaction of the $\mathrm{C} 2 \mathrm{~B}$ domain with membranes are sterically feasible. Owing to the proximity of the binding sites for $\mathrm{Ca}^{2+}$-phosphatidylserine and PIP2, perpendicular binding by $\mathrm{C} 2 \mathrm{~B}$ brings the membranes very close (less than $2 \mathrm{~nm}$, from molecular dynamics simulations). Phosphatidylserine binding by the $\mathrm{Ca}^{2+}$-bound $\mathrm{C} 2 \mathrm{~A}$ domain and electrostatic membrane interactions with other surface-exposed cationic residues of synaptotagmin-1 such as Arg398 and Arg399, which face toward the vesicular membrane in the parallel orientation (Fig. 8c), may act synergistically in the cross-linking of the membranes ${ }^{19,24}$. Finally, in the perpendicular binding mode, several conserved loops perturb the plasma membrane (further discussed in Supplementary Fig. 9), and it is conceivable that under these conditions some stress is exerted on the plasma membrane, which may contribute to a lowering of the energy barrier in the fusion process ${ }^{14}$.

\section{DISCUSSION}

In this study, we found that the polybasic lysine stretch of synaptotagmin-1 interacts independently of $\mathrm{Ca}^{2+}$ with polyanionic PIP2 that is clustered in the membrane by electrostatic interactions with the polybasic juxtamembrane linker region of syntaxin-1A. This interaction can leave the $\mathrm{Ca}^{2+}$-binding sites of synaptotagmin-1 free to bind phosphatidylserine in the vesicle membrane, thus bringing the vesicle membrane very close to the PIP2-syntaxin-1A membrane clusters upon $\mathrm{Ca}^{2+}$ influx. These findings shed new light on previous observations that have been difficult to integrate into a coherent picture. First, $\mathrm{Ca}^{2+}$-independent binding of synaptotagmin-1 to PIP2 positions synaptic vesicles in the vicinity of the acceptor SNAREs before membrane fusion, in agreement with previously reported roles of both synaptotagmin-1 (ref. 43) and PIP2 (reviewed in ref. 44) in docking of synaptic vesicles. Second, because PIP2 binding increases the $\mathrm{Ca}^{2+}$ affinity of the C2B domain more than 40 -fold ${ }^{18}$, prebinding of synaptotagmin-1 to the PIP2 clusters might explain why the $\mathrm{Ca}^{2+}$ sensitivity of exocytosis, for instance in the calyx of Held $(10-25 \mu \mathrm{M})^{45}$, is well below the $K_{\mathrm{d}}$ of synaptotagmin-1 (ref. 13). Indeed, the impaired binding of the KAKA mutant of synaptotagmin-1 to PIP2-enriched syntaxin-1 membrane domains may well explain this mutant's reduced vesicular release probability from hippocampal neurons ${ }^{15}$. Third, our finding sheds new light on the interaction between syntaxin-1A and synaptotagmin-1 that has been a matter of debate (as described in introduction). Our data show that in an intact membrane this interaction is indirect, as it is mediated by syntaxin-bound PIP2. Finally, our results lend further support to the view that synaptotagmin-1 exerts its function in the synapse by regulating the distance between the membranes: upon $\mathrm{Ca}^{2+}$ influx, predocked vesicles are pulled very close to the plasma membrane ( 4-9 $\mathrm{nm}$ by cryo-EM $)^{19,22}$, thus promoting SNARE assembly and triggering fusion. Because cross-linking of the membranes by the $\mathrm{C} 2 \mathrm{~B}$ domain in the perpendicular orientation would result in an $\sim 5$-nm-shorter membrane tethering distance than that of the parallel orientation (from structural considerations) ${ }^{17}$, such a model might explain why disruption of $\mathrm{Ca}^{2+}$ binding to the $\mathrm{C} 2 \mathrm{~A}$ domain (which does not affect perpendicular binding) has only minor effects on release, whereas disruption of the $\mathrm{C} 2 \mathrm{~B}$ domain has much more severe effects on release (reviewed in refs. 2,3). Although our model 
is still speculative, it agrees well with recent in vitro studies by us ${ }^{17}$ and others ${ }^{20}$ showing that membrane fusion is enhanced by orders of magnitude, owing to distance regulation by synaptotagmin-1. Furthermore, it provides a structural explanation for the ability of synaptotagmin to pull plasma and vesicle membranes close together in a $\mathrm{Ca}^{2+}$-dependent manner.

\section{METHODS}

Methods and any associated references are available in the online version of the paper.

Accession code. Coordinates and structure factors have been deposited in the PDB under accession code 2 YOA.

Note: Supplementary information is available in the online version of the paper.

\section{ACKNOWLEDGMENTS}

We thank V. Belov (Max Planck Institute for Biophysical Chemistry, Göttingen, Germany) for the KK114-maleimide dye and D. Cafiso (University of Virginia, Charlottesville, West Virginia, USA) for comments. G.v.d.B. is financed by the Human Frontier Science Program. This work was supported by the US National Institutes of Health (P01 GM072694, to R.J.) and the Deutsche Forschungsgemeinschaft (SFB803). We thank A. Schönle (Max Planck Institute for Biophysical Chemistry, Göttingen, Germany) for the software ImSpector. X-ray diffraction data were collected at beamline X10SA at the Swiss Light Source, and we thank the beamline staff for their help during data collection.

\section{AUTHOR CONTRIBUTIONS}

G.v.d.B., A.H., D.M. and R.J. designed and performed the experiments and wrote the paper. E.I., D.F. and K.K. performed the crystallographic structure determination. H.J.R. and H.G. performed the molecular dynamics simulations. S.M. and U.D. synthesized the peptide. S.W.H., V.M. and C.E. contributed to the microscopy and discussed data. All authors contributed to the manuscript.

\section{COMPETING FINANCIAL INTERESTS}

The authors declare no competing financial interests.

Reprints and permissions information is available online at http://www.nature.com/ reprints/index.html.

1. Südhof, T.C. \& Rothman, J.E. Membrane fusion: grappling with SNARE and SM proteins. Science 323, 474-477 (2009).

2. Rizo, J., Chen, X. \& Araç, D. Unraveling the mechanisms of synaptotagmin and SNARE function in neurotransmitter release. Trends Cell Biol. 16, 339-350 (2006).

3. Chapman, E.R. How does synaptotagmin trigger neurotransmitter release? Annu. Rev. Biochem. 77, 615-641 (2008).

4. Jahn, R. \& Fasshauer, D. Molecular machines governing exocytosis of synaptic vesicles. Nature 490, 201-207 (2012)

5. Sieber, J.J. et al. Anatomy and dynamics of a supramolecular membrane protein cluster. Science 317, 1072-1076 (2007).

6. Aoyagi, K. et al. The activation of exocytotic sites by the formation of phosphatidylinositol 4,5-bisphosphate microdomains at syntaxin clusters. J. Biol. Chem. 280, 17346-17352 (2005)

7. van den Bogaart, G. et al. Membrane protein sequestering by ionic protein-lipid interactions. Nature 479, 552-555 (2011).

8. Choi, U.B. et al. Single-molecule FRET-derived model of the synaptotagmin 1SNARE fusion complex. Nat. Struct. Mol. Biol. 17, 318-324 (2010).

9. Araç, D. et al. Facile detection of protein-protein interactions by one-dimensional NMR spectroscopy. Biochemistry 42, 2774-2780 (2003).

10. Lai, A.L., Huang, H., Herrick, D.Z., Epp, N. \& Cafiso, D.S. Synaptotagmin 1 and SNAREs form a complex that is structurally heterogeneous. J. Mol. Biol. 405, 696-706 (2011).

11. Tang, J. et al. A complexin/synaptotagmin 1 switch controls fast synaptic vesicle exocytosis. Cell 126, 1175-1187 (2006)

12. Xue, M., Ma, C., Craig, T.K., Rosenmund, C. \& Rizo, J. The Janus-faced nature of the C2B domain is fundamental for synaptotagmin-1 function. Nat. Struct. Mol. Biol. 15, 1160-1168 (2008).

13. Radhakrishnan, A., Stein, A., Jahn, R. \& Fasshauer, D. The $\mathrm{Ca}^{2+}$ affinity of synaptotagmin 1 is markedly increased by a specific interaction of its $\mathrm{C} 2 \mathrm{~B}$ domain with phosphatidylinositol 4,5-bisphosphate. J. Biol. Chem. 284, 25749-25760 (2009).

14. McMahon, H.T., Kozlov, M.M. \& Martens, S. Membrane curvature in synaptic vesicle fusion and beyond. Cell 140, 601-605 (2010).
15. Li, L. et al. Phosphatidylinositol phosphates as co-activators of $\mathrm{Ca}^{2+}$ binding to C2 domains of synaptotagmin 1. J. Biol. Chem. 281, 15845-15852 (2006).

16. Schiavo, G., Gu, Q.M., Prestwich, G.D., Söllner, T.H. \& Rothman, J.E. Calciumdependent switching of the specificity of phosphoinositide binding to synaptotagmin. Proc. Natl. Acad. Sci. USA 93, 13327-13332 (1996).

17. van den Bogaart, G. et al. Synaptotagmin-1 may be a distance regulator acting upstream of SNARE nucleation. Nat. Struct. Mol. Biol. 18, 805-812 (2011).

18. van den Bogaart, G., Meyenberg, K., Diederichsen, U. \& Jahn, R. Phosphatidylinositol 4,5-bisphosphate increases the $\mathrm{Ca}^{2+}$ affinity of synaptotagmin-1 40-fold. J. Biol. Chem. 287, 16447-16453 (2012).

19. Araç, D. et al. Close membrane-membrane proximity induced by $\mathrm{Ca}^{2+}{ }^{2+}$ dependent multivalent binding of synaptotagmin-1 to phospholipids. Nat. Struct. Mol. Biol. 13, 209-217 (2006)

20. Hui, E. et al. Mechanism and function of synaptotagmin-mediated membrane apposition. Nat. Struct. Mol. Biol. 18, 813-821 (2011).

21. Herrick, D.Z., Sterbling, S., Rasch, K.A., Hinderliter, A. \& Cafiso, D.S. Position of synaptotagmin I at the membrane interface: cooperative interactions of tandem C2 domains. Biochemistry 45, 9668-9674 (2006).

22. Connell, $E$. et al. Cross-linking of phospholipid membranes is a conserved property of calcium-sensitive synaptotagmins. J. Mol. Biol. 380, 42-50 (2008).

23. Kim, J.Y. et al. Solution single-vesicle assay reveals $\mathrm{PIP}_{2}$-mediated sequential actions of synaptotagmin-1 on SNAREs. EMBO J. 31, 2144-2155 (2012).

24. Kuo, W., Herrick, D.Z. \& Cafiso, D.S. Phosphatidylinositol 4,5-bisphosphate alters synaptotagmin 1 membrane docking and drives opposing bilayers closer together. Biochemistry 50, 2633-2641 (2011).

25. Joung, M.J., Mohan, S.K. \& Yu, C. Molecular level interaction of inositol hexaphosphate with the $\mathrm{C} 2 \mathrm{~B}$ domain of human synaptotagmin I. Biochemistry 51 , 3675-3683 (2012).

26. Fernandez, I. et al. Three-dimensional structure of the synaptotagmin 1 C2B-domain: synaptotagmin 1 as a phospholipid binding machine. Neuron 32 1057-1069 (2001).

27. Vrljic, M. et al. Post-translational modifications and lipid binding profile of insect cell-expressed full-length mammalian synaptotagmin 1. Biochemistry $\mathbf{5 0}$, 9998-10012 (2011).

28. Eggeling, C. et al. Direct observation of the nanoscale dynamics of membrane lipids in a living cell. Nature 457, 1159-1162 (2009).

29. Mueller, V. et al. STED nanoscopy reveals molecular details of cholesteroland cytoskeleton-modulated lipid interactions in living cells. Biophys. J. 101 1651-1660 (2011).

30. Milosevic, I. et al. Plasmalemmal phosphatidylinositol-4,5-bisphosphate leve regulates the releasable vesicle pool size in chromaffin cells. J. Neurosci. 25 2557-2565 (2005).

31. Rickman, C. et al. Synaptotagmin interaction with the syntaxin/SNAP-25 dimer is mediated by an evolutionarily conserved motif and is sensitive to inosito hexakisphosphate. J. Biol. Chem. 279, 12574-12579 (2004).

32. Jensen, M.H., Morris, E.J. \& Simonsen, A.C. Domain shapes, coarsening, and random patterns in ternary membranes. Langmuir 23, 8135-8141 (2007).

33. Murray, D. et al. Electrostatic properties of membranes containing acidic lipids and adsorbed basic peptides: theory and experiment. Biophys. J. 77, 3176-3188 (1999).

34. Levental, I. et al. Calcium-dependent lateral organization in phosphatidylinositol 4,5-bisphosphate (PIP2)- and cholesterol-containing monolayers. Biochemistry 48 8241-8248 (2009).

35. Ausili, A., Corbalán-García, S., Gómez-Fernández, J.C. \& Marsh, D. Membrane docking of the $\mathrm{C} 2$ domain from protein kinase $\mathrm{C} \alpha$ as seen by polarized ATR-IR. The role of PIP 2 . Biochim. Biophys. Acta 1808, 684-695 (2011).

36. Lai, C.L., Landgraf, K.E., Voth, G.A. \& Falke, J.J. Membrane docking geometry and target lipid stoichiometry of membrane-bound $\mathrm{PKC} \alpha \quad \mathrm{C} 2$ domain: a combined molecular dynamics and experimental study. J. Mol. Biol. 402, 301-310 (2010).

37. Chen, C.H. et al. Configuration of PKC $\alpha-C 2$ domain bound to mixed SOPC/SOPS lipid monolayers. Biophys. J. 97, 2794-2802 (2009).

38. Montaville, P. et al. The PIP2 binding mode of the $\mathrm{C} 2$ domains of rabphilin-3A. Protein Sci. 17, 1025-1034 (2008).

39. Guerrero-Valero, M. et al. Structural and mechanistic insights into the association of PKC $\alpha$-C2 domain to PtdIns(4,5)P2. Proc. Natl. Acad. Sci. USA 106, 6603-6607 (2009).

40. Verdaguer, N., Corbalan-Garcia, S., Ochoa, W.F., Fita, I. \& Gómez-Fernández, J.C. $\mathrm{Ca}^{2+}$ bridges the $\mathrm{C} 2$ membrane-binding domain of protein kinase $\mathrm{C} \alpha$ directly to phosphatidylserine. EMBO J. 18, 6329-6338 (1999).

41. Cheng, $\mathrm{Y}$. et al. Crystallographic identification of $\mathrm{Ca}^{2+}$ and $\mathrm{Sr}^{2+}$ coordination sites in synaptotagmin I C2B domain. Protein Sci. 13, 2665-2672 (2004).

42. Marrink, S.J., Risselada, H.J., Yefimov, S., Tieleman, D.P. \& de Vries, A.H. The MARTINI forcefield: coarse grained model for biomolecular simulations. J. Phys. Chem. B 111, 7812-7824 (2007).

43. de Wit, H. et al. Synaptotagmin-1 docks secretory vesicles to syntaxin-1/SNAP-25 acceptor complexes. Cell 138, 935-946 (2009).

44. Martin, T.F.J. Role of $\mathrm{PI}(4,5) \mathrm{P}_{2}$ in vesicle exocytosis and membrane fusion. Subcell. Biochem. 59, 111-130 (2012).

45. Schneggenburger, R. \& Neher, E. Presynaptic calcium and control of vesicle fusion. Curr. Opin. Neurobiol. 15, 266-274 (2005). 


\section{ONLINE METHODS}

Synaptotagmin purification. DNA constructs of full-length synaptotagmin-1 and mutant C2AB fragments (rat synaptotagmin-1 ${ }_{97-421}$ ) were as described ${ }^{13,17}$, except for the R398Q R399Q (RQRQ) mutant, for which we ordered a synthetic gene (Genscript) in the NdeI-EcoRI site of pET28a(+) (sequence in Supplementary Note). Proteins were expressed in Escherichia coli strain BL21CodonPlus (DE3)-RIPL (Stratagene) and purified with $\mathrm{Ni}^{2+}$-affinity chromatography followed by ion-exchange chromatography as described ${ }^{13,17,18}$. The single-cysteine mutant of C2AB (C278S S342C) was labeled by 20-min incubation with a 2 - to 10 -fold molar excess of Alexa Fluor $488 \mathrm{C}_{5}$-maleimide (Invitrogen) or KK114-maleimide ${ }^{46}$ in $50 \mathrm{mM}$ HEPES, pH 7.4, $500 \mathrm{mM} \mathrm{NaCl}$ and $100 \mu \mathrm{M}$ Tris(2-carboxyethyl)phosphine. The labeled protein was separated from the free dye by size-exclusion chromatography (Sephadex G50, Sigma).

Antibody labeling. We labeled $250 \mu \mathrm{l}$ of a $0.2 \mathrm{mg} / \mathrm{ml}$ concentration of the antibody HPC-1 (to syntaxin-1; Sigma-Aldrich, S0664) ${ }^{47}$ with $\sim 250 \mathrm{nmol}$ Alexa Fluor $488 \mathrm{C}_{5}$-maleimide (Invitrogen) and $100 \mu \mathrm{l}$ of $1 \mathrm{mg} / \mathrm{ml}$ antibody 41.1 (to synaptotagmin-1; Synaptic Systems, 105011) with $5 \mu \mathrm{l}$ of $\sim 10 \mathrm{mM}$ Atto647N NHS-ester (Atto-tec) by 20-min incubation in $10 \mathrm{mM}$ Na phosphate, $\mathrm{pH} 7.6$, with $250 \mathrm{mM}$ $\mathrm{NaCl}$ followed by size-exclusion chromatography (Sephadex G50) for removal of unreacted dye. The size-exclusion chromatography step resulted in an $\sim 5$-fold dilution of the antibody concentration.

Peptide synthesis. Synthesis of Atto647N (Atto-tec)-labeled syntaxin-1A 257-288 was as described ${ }^{7}$, and syntaxin-1 $\mathrm{A}_{260-265}$ (KARRKK) was synthesized by solid-phase peptide synthesis on Fmoc-Lys(Boc)-rink amide resin following standard Fmoc strategies. Purification was performed by HPLC on C18 material, using water and acetonitrile as mobile phases. The peptide identity was confirmed by MS.

Cell culture and immunofluorescence. PC12 cells (clone 251) ${ }^{48}$ were maintained and propagated as described ${ }^{5}$. Transfection was with Lipofectamine-LTX, as described ${ }^{7}$. Membrane sheets were generated as described ${ }^{5,7}$, except that the PC12 buffer contained no EDTA (20 mM K HEPES, pH 7.4, 120 mM K gluconate, $20 \mathrm{mM} \mathrm{K}$ acetate, $2 \mathrm{mM}$ ATP and $0.5 \mathrm{mM}$ DTT). All experiments with PC1 2 cells were carried out in this buffer. Immunostaining of unfixed membrane sheets was performed by blocking for 5 min with 3\% (w/v) BSA in PC12 buffer and incubation with $2 \mu \mathrm{g} / \mathrm{ml}$ Alexa Fluor 488-labeled HPC-1 antibody for $<5$ min followed by brief washing. For blocking endogenous synaptotagmin-1, the PC12 membrane sheets were incubated with $4 \mu \mathrm{g} / \mathrm{ml}$ Atto647N-labeled 41.1 antibody for $30 \mathrm{~min}$. PC12 membranes were labeled with Atto647N PE, Atto647N PC $^{28,29}$, BODIPY-FL PC or Top-Fluor PIP2 (Avanti) by 5-min incubation with $1 \mu \mathrm{M}$ of BSA-complexed lipid analog as described ${ }^{28,29}$. C2AB was used at a final concentration of $100 \mathrm{nM}$ for the incubation of membrane sheets or of artificial membranes. Liposomes were used at a final lipid concentration of $\sim 50 \mu \mathrm{M}$ (about 5-nM liposome concentration ${ }^{17,49}$ ).

Liposome preparation. Liposomes were prepared with a 5:2:1:1 molar ratio of DOPC/DOPE/DOPS/cholesterol with 1\% BODIPY-FL PC as described ${ }^{17}$. Briefly, the lipid mixture was dried in a rotary evaporator and resuspended to a total lipid concentration of $30 \mathrm{mM}$ in $20 \mathrm{mM}$ HEPES, pH 7.4, $150 \mathrm{mM} \mathrm{KCl}$ with $1.5 \% \mathrm{Na}^{+}$cholate. Liposomes were then formed by size-exclusion chromatography (Sephadex G50) in the same buffer without $\mathrm{Na}^{+}$cholate. Full-length synaptotagmin-1 was reconstituted in the liposomes at a protein/lipid molar ratio of $1: 1,000$ as described ${ }^{17}$.

Supported lipid bilayers. Supported lipid bilayers were generated by spin-coating (at 2,000 r.p.m.) a $3 \mathrm{mg} / \mathrm{ml}$ solution in chloroform of the required cholesterol, DOPC, DOPS and brain PIP2 (all from Avanti) mixture with $0.1 \mathrm{~mol} \%$ Atto647N phosphatidylethanolamine or BODIPY-FL PC on glass ${ }^{50}$. In case syntaxin$1 \mathrm{~A}_{257-288}$ was present, the lipids were dissolved to $1 \mathrm{mg} / \mathrm{ml}$ in methanol and mixed to a final protein/lipid ratio of approximately 1:100 of unlabeled peptide and 1:1,000 of Atto647N-labeled peptide in 2,2,2-trifluoroethanol (TFE), followed by spin-coating. This procedure gives 1-3 stacked supported lipid bilayers $^{50}$. Microscopy experiments were performed in 20 mM HEPES, pH 7.4, with $150 \mathrm{mM} \mathrm{KCl}$. $\mathrm{C} 2 \mathrm{AB}$ and liposome binding assays were performed identically to the experiments with PC12 membrane sheets.
Confocal and STED microscopy. Confocal and STED imaging and STED-FCS were performed on a home-built confocal STED microscope that was described previously in detail ${ }^{29}$ except that the setup was expanded with a fast beam scanner (Yanus, Till-Photonics) and a blue excitation ( $~ 80$ ps pulse width, LDH-P-485B, PicoQuant) and STED laser (577 nm OPSL CW, Coherent) for fast STED imaging and STED-FCS of red-emitting (KK114 and Atto647N) and blue-emitting (Alexa Fluor 488) dyes. The excitation intensity was in the range of $5-8 \mu \mathrm{W}$ at a diffraction-limited focal diameter of approximately $250 \mathrm{~nm}(640 \mathrm{~nm})$ and $190 \mathrm{~nm}(485 \mathrm{~nm})$. The average STED power was $180 \mathrm{~mW}(770 \mathrm{~nm})$ or $120 \mathrm{~mW}$ $(577 \mathrm{~nm})$, measured in the back aperture of the objective. A software-gated detection scheme was used in the STED recordings using the blue continuous-wave STED laser ${ }^{51}$. For imaging, the resolution was approximately $50 \mathrm{~nm}$ (for both the 770-nm and $577-\mathrm{nm}$ STED channels), as measured independently by imaging 20-nm-sized fluorescent beads.

Details of the STED-FCS analysis are as described ${ }^{28,29}$. Briefly, we first recorded FCS data for different STED intensities, that is, for observation areas of different diameters. Fitting the FCS data as described ${ }^{28,29}$ resulted in the dependence of the average apparent diffusion coefficient $D$ on the size of the observation area $d$. The diameter $d$ of the observation area for each STED intensity was calibrated by STED-FCS measurements of a KK114-labeled phosphatidylethanolamine freely diffusing in a DOPC supported lipid bilayer.

Purification and crystallization of the C2B fragment. For expression of the $\mathrm{C} 2 \mathrm{~B}$ fragment, we ordered a synthetic gene (Genscript) in the NdeI-XhoI restriction sites of pET-28a(+). This gene encodes residues $271-421$ of rat synaptotagmin-1 (Swiss-Prot P21707). The codon usage was optimized for expression in Escherichia coli (strain K12) (sequence in Supplementary Note). E. coli strain BL21(DE3) expressing C2B was grown in Luria Broth at $37^{\circ} \mathrm{C}$ to an optical density of 0.6 at $600 \mathrm{~nm}$, induced with $1 \mathrm{mM} \mathrm{IPTG}$ and incubated at $30^{\circ} \mathrm{C}$ for $4 \mathrm{~h}$. The cells were harvested and resuspended in buffer A (25 mM HEPES, pH 7.4, $100 \mathrm{mM} \mathrm{NaCl})$. Bacteria were lysed by sonication. Cell debris was removed by centrifugation $(5,000 \mathrm{~g}$ for $20 \mathrm{~min})$, and the supernatant was incubated with Ni-NTA beads for $1 \mathrm{~h}$ at $4^{\circ} \mathrm{C}$. The beads were washed with buffer A containing $30 \mathrm{mM}$ imidazole, and the protein was eluted with buffer A containing $300 \mathrm{mM}$ imidazole. The protein was then diluted in a 1:1 ratio with buffer A supplemented with $5 \mathrm{mM} \mathrm{CaCl}_{2}$. Precipitant was removed by centrifugation. The protein was then concentrated and applied onto a Superdex-75 16/60 (GE Healthcare) column. Running buffer was buffer A with $5 \mathrm{mM} \mathrm{CaCl}_{2}$. The protein was concentrated to $30 \mathrm{mg} / \mathrm{ml}$, aliquoted and then frozen in liquid nitrogen and stored at $-80^{\circ} \mathrm{C}$. A $100-\mathrm{mM}$ phosphoserine (TCI Europe) stock solution was prepared with water and adjusted to $\mathrm{pH} 6$ with $\mathrm{NaOH}$. Crystals were grown at $20^{\circ} \mathrm{C}$ with the hanging-drop vapor-diffusion method. Two microliters of $16 \mathrm{mg} / \mathrm{ml} \mathrm{C2B} \mathrm{in} 0.1 \mathrm{M} \mathrm{NaCl}, 25 \mathrm{mM}$ HEPES, $\mathrm{pH} 7.4$, $5 \mathrm{mM} \mathrm{CaCl}_{2}$ and $20 \mathrm{mM}$ phosphoserine were mixed with $2 \mu \mathrm{l}$ of the precipitant containing $0.2 \mathrm{M} \mathrm{KSCN}$ and 20\% PEG 600. Crystals were transferred into a cryoprotectant consisting of 10\% ethylene glycol, 26\% PEG 600, 0.1 M KSCN, 5 mM $\mathrm{CaCl}_{2}$ and $20 \mathrm{mM}$ phosphoserine and then flash cooled in liquid nitrogen.

$\mathrm{X}$-ray data collection and structure determination. Diffraction data were collected at a wavelength of $1.0 \AA$ at $100 \mathrm{~K}$ on beamline X10SA at the Swiss Light Source (Paul Scherrer Institute, Villigen, Switzerland). Data were processed with $\mathrm{XDS}^{52}$. Crystals diffracted to $1.5-\AA$ resolution and belonged to the tetragonal space group $P 42_{1} 2$. There were two molecules in the asymmetric unit, which were related by pseudotranslational noncrystallographic symmetry (pseudotranslation vector: $u=0, v=0, z=0.5)$. The structure was determined by molecular replacement by using BALBES ${ }^{53}$ with PDB $1 \mathrm{UOV}^{41}$ as a search model. The structure was refined with REFMAC5 (ref. 54), and model building was done with COOT ${ }^{55}$. The final model comprises residues 271-418 for molecule A and residues 271-419 for molecule B. The structure was analyzed with PROCHECK ${ }^{56}$, and $90.9 \%$ of residues were located in the core region of the Ramachandran plot and $9.1 \%$ in the allowed region. Figures were prepared with PyMol (http://www.pymol.org/).

Molecular dynamics. The molecular dynamics simulations were performed with the GROMACS simulation package ${ }^{57}$ and are described in the Supplementary Note.

46. Kolmakov, K. et al. Red-emitting rhodamine dyes for fluorescence microscopy and nanoscopy. Chemistry. 16, 158-166 (2010). 
47. Barnstable, C.J., Hofstein, R. \& Akagawa, K. A marker of early amacrine cell development in rat retina. Brain Res. 352, 286-290 (1985).

48. Heumann, R., Kachel, V. \& Thoenen, H. Relationship between NGF-mediated volume increase and "priming effect" in fast and slow reacting clones of PC12 pheochromocytoma cells. Role of cAMP. Exp. Cell Res. 145, 179-190 (1983).

49. van den Bogaart, G. et al. One SNARE complex is sufficient for membrane fusion. Nat. Struct. Mol. Biol. 17, 358-364 (2010).

50. Mennicke, U. \& Salditt, T. Preparation of solid-supported lipid bilayers by spincoating. Langmuir 18, 8172-8177 (2002).

51. Vicidomini, G. et al. Sharper low-power STED nanoscopy by time gating. Nat. Methods 8, 571-573 (2011).

52. Kabsch, W. Automatic processing of rotation diffraction data from crystals of initially unknown symmetry and cell constants. J. Appl. Crystallogr. 26, 795-800 (1993).
53. Long, F., Vagin, A.A., Young, P. \& Murshudov, G.N. BALBES: a molecular-replacement pipeline. Acta Crystallogr. D Biol. Crystallogr. 64, 125-132 (2008).

54. Murshudov, G.N. Vagin, A.A \& Dodson, E.J. Refinement of macromolecular structures by the maximum-likelihood method. Acta Crystallogr. D Biol. Crystallogr. 53, 240-255 (1997).

55. Emsley, P. \& Cowtan, K. COOT: model-building tools for molecular graphics. Acta Crystallogr. D Biol. Crystallogr. 60, 2126-2132 (2004).

56. Laskowski, R.A., MacArthur, M.W., Moss, D.S. \& Thornton, J.M. PROCHECK a program to check the stereochemical quality of protein structures. J. Appl. Crystallogr. 26, 283-291 (1993).

57. Hess, B., Kutzner, C., van der Spoel, D. \& Lindahl, E. GROMACS 4: algorithms for highly efficient, load-balanced, and scalable molecular simulation. J. Chem. Theory Comput. 4, 435-447 (2008). 